\title{
Endoscopic posterior cordotomy for treatment of dyspnea due to vocal fold immobility
}

\author{
Narin Nard Carmel-Neiderman, Max Chason, Anat Wengier, Oshri Wasserzug, Oren Cavel, Gilad Horowitz, Barak \\ Ringel, Anton Warshavsky, Yael Oestreicher-Kedem
}

Department of Otolaryngology Head and Neck Surgery and Maxillofacial Surgery, Tel Aviv Sourasky Medical Center, affiliated to the Sackler Faculty of Medicine, Tel Aviv University, Tel Aviv, Israel

\begin{abstract}
Introduction: Several surgical procedures have been described for the treatment of respiratory distress secondary to vocal fold immobility (VFI), but the contribution of posterior cordotomy (PC) to tracheostomy weaning or prevention has not been studied in depth, particularly in the acute setting. The objective of this study was to show the effectiveness of PC to relieve dyspnea, prevent the need for tracheostomy, and enable decannulation in patients with VFI.

Methods: We conducted a retrospective study and reviewed the medical records of all patients whose dyspnea warranted surgical intervention from January 2013 to January 2018. Data were retrieved on epidemiology, etiology, and duration of VFI, tracheostomy dependence, success in decannulation from tracheostomy or respiratory relief, number of procedures until decannulation, and complications.

Results: Twelve suitable patients were identified of whom eleven had bilateral VFI and one had unilateral VFI. Five were tracheostomy-dependent. Ten patients underwent unilateral PC, and two patients underwent bilateral PC. All the patients experienced respiratory relief, eleven after a single PC and one after two PCs. All tracheostomy-dependent patients were decannulated. The mean follow-up after PC was 24.55 months during which none of the patients required a re-tracheostomy and three patients required revision of the PC. There were no surgical complications. Postoperatively, eight patients (67\%) experienced a breathy voice and three patients $(25 \%)$ had dysphagia for fluids. No patient had aspiration pneumonia.

Conclusions: We conclude that PC is an easy, safe, and effective procedure for tracheostomy weaning and respiratory relief in patients with VFI. A revision PC may be indicated in some patients. A breathy voice is to be expected, and a few patients will experience dysphagia to fluids that may be addressed by instructing the patient to use a fluid thickener and take small sips.
\end{abstract}

Key words: Vocal fold immobility; posterior cordotomy; dyspnea; tracheostomy.

Correspondence: Yael Oestreicher-Kedem, Department of Otolaryngology, Head and Neck Surgery and Maxillofacial Surgery, Tel Aviv Sourasky Medical Center, 6 Weizman St., Tel Aviv, 6423906 Israel.

Tel. +972.52.6212179 - Fax: +972.3.6052905. E- mail: dkyo@013.net

Contributions: All the authors made a substantive intellectual contribution, have read and approved the final version of the manuscript and agreed to be accountable for all aspects of the work.

Conflict of interest: The authors declare that they have no competing interests. The Alma Pixel $\mathrm{CO}_{2}$ laser was on loan to our department from Alma Lasers.

Availability of data and materials: The datasets used and/or analyzed during the current study are available from the corresponding author on reasonable request.

Ethics approval and consent to participate: All procedures performed were in accordance with the ethical standards of the Institutional Research Committee and with the 1964 Helsinki declaration and its latest amendment. The study was approved by the institutional Helsinki board (008-18-TLV).

Consent for publication: Not applicable. 


\section{Introduction}

Bilateral vocal fold immobility (VFI) is a serious disability, and about $40 \%$ of patients with VFI will experience acute respiratory distress necessitating a tracheostomy [1]. Despite the fact that most patients will eventually regain VF motion and experience relief of their dyspnea, $19 \%$ of the patients will remain tracheostomy-dependent. Permanent tracheostomy is associated with a decline in quality of life [2], and most patients with bilateral VFI and dyspnea will prefer to avoid it if at all possible.

Bilateral VFI may be due to either a neural injury, such as bilateral recurrent laryngeal nerve palsy, or to a mechanical injury to the posterior glottis, such as posterior glottic stenosis (PGS) that prevents arytenoid motion and VF abduction. The most common causes of bilateral vocal cord paralysis due to recurrent laryngeal nerve injury are: surgical trauma (44\%), malignancies in the neck base of skull or upper mediastinum $(17 \%)$, neurological disease $(12 \%)$ and idiopathic $(12 \%)$ [1,3]. The most common iatrogenic cause of bilateral VFI is total thyroidectomy, comprising 10.7$78.6 \%$ of cases of bilateral VFI in various case series [4]. Causes of posterior glottic stenosis included: iatrogenic trauma $(55 \%)$, idiopathic $(18.5 \%)$, autoimmune $(18.5 \%)$, and other traumas $(8 \%)$ [4]. Prolonged intubation is the leading cause $(15 \%)$ of iatrogenic VF fixation [1]. Interestingly, while the rate of iatrogenic post-thyroidectomy bilateral VFI has decreased in recent years, the rate of bilateral VFI due to posterior glottic stenosis post-intubation or prolonged mechanical ventilation $(26.7-42.8 \%)$ has remained steady and possibly even increased [4].

Various surgical procedures have been suggested to improve dyspnea in patients with bilateral VFI, including tracheostomy, arytenoidectomy (partial or complete, endoscopic or transcervical), cordopexy, and endoscopic posterior cordotomy (PC). These methods allow for a passive increase in the posterior glottic space. Laryngeal reinnervation and electrical laryngeal pacing of the paralyzed VFs are two techniques which aim to restore VF motion and active abduction. They both attempt to regain movement of the paralyzed VF and synchronize it with breathing, swallowing, and speech. Reinnervation requires high levels of surgical training, it is an invasive surgery, and recovery takes months. Electrical pacing, on the other hand, is experimental, also requires a high level of surgical training, and necessitates surgical instruments that are not commonly available. Consequently, these two methods are not yet suitable for immediate relief of dyspnea in patients with bilateral VFI.

Endoscopic PC by means of a $\mathrm{CO}_{2}$ laser was introduced by Dennis and Kashimna [5]. They described the resection of a 4-mm segment of the posterior aspect of the paralyzed VF from the vocal process of the arytenoid anteriorly and laterally to the false VF in the shape of the letter "C". This resection enlarges the posterior glottic gap by around $6 \mathrm{~mm}$. PC is a simple procedure that is very easy to perform, does not require sophisticated equipment, and, most importantly, it provides instant relief of dyspnea in patients with VFI. Moreover, it can be repeated if necessary. The major downside is the permanent VF damage that may lead to dysphonia and dysphagia and aspirations due to glottic insufficiency.

The purpose of our study was to examine the efficacy and safety of PC to relieve dyspnea, prevent the need for a tracheostomy, and enable decannulation in patients with VFI.

\section{Materials and Methods}

This was a retrospective cohort study at Tel Aviv Sourasky Medical Center, a tertiary referral center in Israel. The medical records of all patients who underwent PC due to dyspnea from January 2013 to January 2018 were reviewed. Data were retrieved on epidemiology, etiology of VFI and its duration, tracheostomy dependence, success in decannulation from tracheostomy or respiratory relief, number of procedures until decannulation, complications associated with the procedure, post opertive voice evaluation using the Grade, Roughness, Breathiness, Asthenia, Strain (GRABS) [5]. scale and patients' report on dysphagia and dysphonia at the follow up visits. The data were collected on Excel files and then underwent statistical analysis with SPSS. The study was approved by the Institutional Helsinki Board (008-18-TLV).

\section{Posterior cordotomy technique}

All PCs were done under general anesthesia. Patients were ventilated through a $5-\mathrm{mm}$ microlaryngeal endotracheal tube or through an existing tracheostomy. They were administered intravenous steroids (dexamethasone) and antibiotics (cefazolin) before the procedure. Direct laryngoscopy and suspension were performed, and a microscope was used to visualize the glottis. $\mathrm{A} \mathrm{CO}_{2}$ laser (Lumenis Sharplan $40 \mathrm{c} \mathrm{CO}_{2}$ or Alma Pixel $\mathrm{CO}_{2}$ ) with micromanipulator in supra-pulse and continuous mode at 4-6 watts was used to perform the PC. The PC cut was begun at the medial side of the paralyzed VF anterior to the vocal process and continued laterally through all the layers of the VF (mucosa, lamina propria, and muscle) through the ventricle and the false VF until the inner aspect of the thyroid cartilage. The intubated patients were extubated at the end of the procedure. Tracheostomy-dependent patients were weaned by gradually during the weeks following surgery, downsizing the tracheostomy tube until closure of the tracheostomy tube became feasible. The tracheostomy tube was then removed under medical supervision. All patients were hospitalized 5-7 days posteoperatively at the otolaryngology department. After discharge patients were followed at the otolaryngology outpatient clinic at scheduled intervals beginning one month postoperatively. Patients were instructed to approach the emergency department if they experience worsening dyspnea.

\section{Results}

Between January 2013 and January 2018, 20 patients with respiratory distress due to impaired VF mobility presented at the Voice and Swallowing Clinic at Tel Aviv Medical Center. Seven patients were tracheostomy-dependent, 10 reported respiratory distress which impaired their daily activities, and 3 reported minor respiratory distress that did not impair their daily activities (Table 1). PC was offered to the 13 (e.g. patients who exhibited respiratoty distress or were tracheostomy dependent). A PC was not offered to patients who exhibited only mild respiratory distress $(n=3)$ or to

Table 1. Etiology and extent of vocal fold immobility causing airway compromise.

Etiology Unilateral Bilateral

Prolonged mechanical ventilation 6 causing posterior glottic stenosis

\begin{tabular}{lcc} 
Thyroidectomy & 4 & 4 \\
\hline $\begin{array}{l}\text { Radiotherapy to the larynx } \\
\text { Heart surgery }\end{array}$ & 1 & 3 \\
\hline $\begin{array}{l}\text { Pemphigus vulgaris } \\
\text { Idiopathic }\end{array}$ & 1 \\
Total & 5 & 1 \\
\hline
\end{tabular}


patients $(n=4)$ with contraindications to PC including a history of recurrent aspirations $(n=2)$, combined supraglottic and glottic stenosis $(n=1)$ or impaired pulmonary function $(n=1)$. A total of 12 patients consented to PC and were included in the study (Table 2) 11 with bilateral VFI and one with unilateral VFI. This single patient with unilateral VFI exhibited dyspnea and restricted glottic opening, despite only unilateral VFI. Five patients were tracheostomy dependent wishing to wean from the tracheostomy.

Ten patients underwent unilateral $\mathrm{PC}$ and two underwent bilateral PC (Figure 1). Eleven patients underwent a PC once and achieved adequate respiratory improvement and one patient required revision of the PC (1 week later). Other glottic narrowing pathologies were addressed during the PC: two patients underwent incision of a posterior web, one patient underwent incision of an anterior web and keel placement, and one patient underwent posterior resection of an edematous contralateral false VF. No complications were noted during the PC. Five patients with impending airway obstruction underwent $\mathrm{PC}$ as an urgent procedure due to acute increasing respiratory distress and in order to avoid an urgent tracheostomy. All patients in this group had a history of long-term VFI with progressive dyspnea that eventually required urgent surgical intervention due to increased airway compromise. All five patients chose to undergo a PC over a tracheostomy. The etiologies for VFI in this group were thyroidectomy, Charcot-Marie-Tooth disease, prolonged intubation, and radiotherapy to the larynx. All patients experienced relief of their respiratory distress, and the five patients who were tracheostomy-dependent were successfully decannulated. Time to decannulation ranged between 1-11 months (mean 6 months).

The patients were followed up for $24.55 \pm 19.20$ months (range 2-56 months). Three patients required a revision PC during the follow up period. One had PGS and experienced recurrent episodes of stridor, which were attributed to re-stenosis of the posterior glottis, during the 3 months following the PC. He underwent revision of the PC 3 months after the first one and was dyspnea free at the last follow up 53 months later. A second patient had dyspnea that impaired her daily activity due to bilateral VFP post total thyroidectomy. She underwent PC and her dyspnea was releaved. Her dyspnea recurred 32 months post initial PC. She underwent a second PC and was dyspnea free at the last follow up 5 months later. The third patient was tracheostomy dependent due to bilateral VFI after total thyroidectomy. She underwent unilateral PC and was decannulated. Three years after the initial PC she developed progressive dyspnea due to closure of the PC. She then underwent a second PC, which relieved her dyspnea for 2 months, after which it recurred. She underwent a third PC in combination with suture lateralization of one VF, and she was symptom free for 16 months. In this patient suture lateralization was chosen over bilateral PC since she had slight movement of the contralateral arytenoid and to keep one side of the laryngeal anatomy intact for future possible reinnervation procedures. Fifteen months after the the third PC and unilateral suture lateralization her dyspnea recurred and she underwent endoscopic partial arytenoidectomy and revision of the suture lateralization. She was dyspnea-free at the last follow up 3 months later. One patient with PGS who was successfully decannulated suffered cardiac arrest due to hypoglycemia 11 months following decannulation: he was resuscitated, intubated, and later re-tracheostomized. All 12 patients were satisfied by the respiratory relief that had been provided by the PC, and they all resumed a per-oral diet. We did not find any difference in relief of respiratory distress after PC, independent of the etiology of VFI (paralysis or fixation). Three patients $(25 \%)$ reported cough during fluid intake, and they were instructed to use a fluid thickener and take small sips. None of the patients experienced aspiration pneumonia. Eight patients $(67 \%)$ reported a breathy voice. The average postoperative Grade, Roughness, Breathiness, Asthenia, Strain (GRABS) scale [5,6] was G1.75R1.37A1.28B1.87S1, indicating an average voice quality.

Table 2. Characteristics of the posterior cordotomy patient population.

\begin{tabular}{lc}
\hline Number of patients & 12 \\
Male sex (\%) & $8(66)$ \\
\hline Mean age, years (SD) & $57.6(20)$ \\
History of tobacco use (\%) & $5(42)$ \\
\hline Bilateral VFI (\%) & $11(92)$ \\
PGS (\%) & $6(50)$ \\
Etiology of dyspnea & \\
VFI after thyroidectomy & 5 \\
$\quad$ VFI due to Charcot-Marie-Tooth disease & 1 \\
PGS after prolonged mechanical ventilation & 5 \\
PGS after radiotherapy to the larynx & 1 \\
Tracheostomy dependency (\%) & $5(42)$ \\
Duration range, years (mean) & $1-10(5.5)$ \\
\hline
\end{tabular}

SD, standard deviation; PGS, posterior glottic stenosis; VFI, vocal fold immobility.
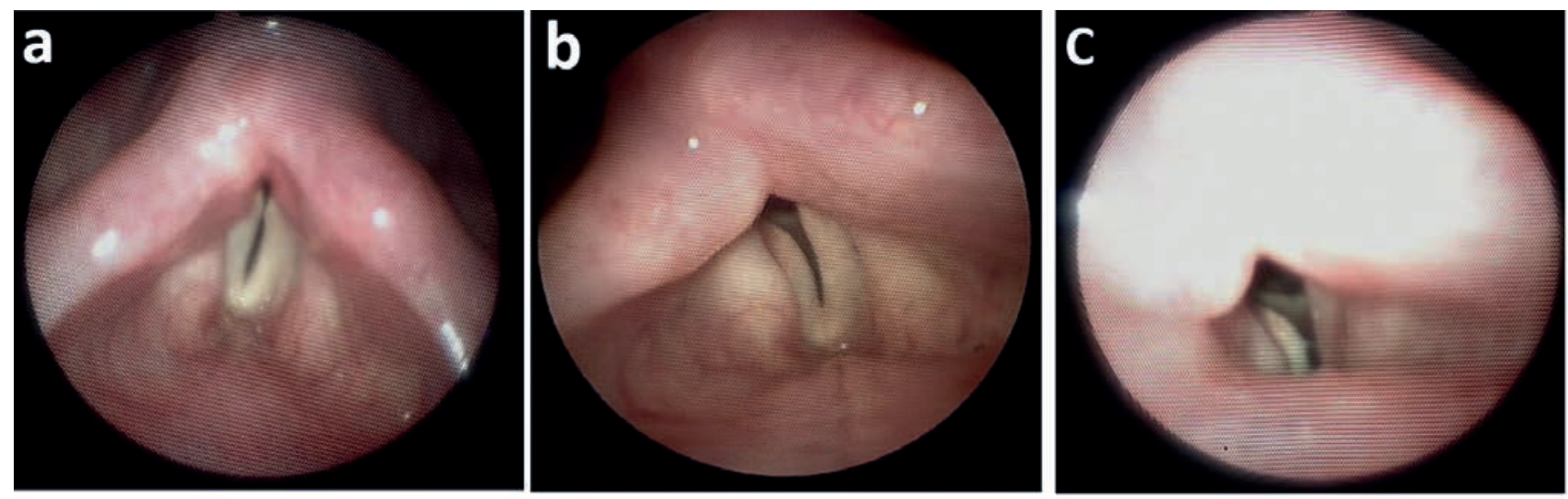

Figure 1. a) Image of the glottis of a patient with bilateral vocal fold immobility in maximal inspirium. b) Same patient after right posterior cordotomy. c) same patient after revision right posterior cordotomy and suture lateralization. 


\section{Discussion}

A PC is a procedure with many advantages. It is quick and technically simple to perform, it produces immediate relief of dyspnea, and it has a low risk of complications. If needed, re-PC can be performed to further alleviate the dyspnea [7]. All patients of our study had a positive outcome with regard to relieving their respiratory distress. We also achieved complete decannulation among all the patients who were tracheostomy-dependent, and only two patients required a second PC. One patient required re-tracheostomy after an episode of recurrent prolonged mechanical ventilation. Our rate of decannulation $(100 \%)$ was similar to that reported by others $(83-$ $100 \%$ ) [9-11] although our cohort included $42 \%$ of tracheostomydependent patients. In other series [9-11] the rate of tracheostomy dependent patients ranged from $7.6 \%$ to $57.1 \%$.

The classic approach to impending airway obstruction in patients with VFI is an urgent tracheostomy. We showed that a PC may replace a tracheostomy, which many patients prefer to avoid, in the setting of impending airway in patients with long-standing VFI. Five of our study patients with impending airway obstruction underwent $\mathrm{PC}$ as an urgent procedure due to acute respiratory distress and to avoid undergoing an urgent tracheostomy.

One disadvantage of PC is that voice quality is not always ideal due to a posterior glottis gap created by the procedure. Most our patients reported a breathy voice postoperatively, and the average GRABS score reflected G1.75R1.37A1.28B1.87S1, indicating an average voice quality. However, when asked on clinic visits about their voice quality, overall, the patients were satisfied by the surgical results, possibly because their preoperative voice quality was already impaired and did not worsen to an extent that influenced their judgment. We did not have sufficient preoperative data on voice quality for comparison as $41.2 \%(n=5)$ of the patients were hospitalized and treated urgently. Hillel et al. reported that a PC may even improve voice quality in patients with bilateral vocal cord paralysis [12]. Another disadvantage of a PC is the risk of aspiration pneumonia due to the posterior glottis gap [10]. None of our patients had history of aspiration pneumonia postoperatively despite the fact that three of them reported cough on fluid intake. A PC may scar over time and a recurrent glottic narrowing can develop and cause a recurrent airway compromise. This may be addressed by revision of the PC or by other glottis-widening procedures, such as suture lateralization, arytenoidectomy, or proceeding with a tracheostomy. The rate of revision of the $\mathrm{PC}$ varies in published series and ranges between 6-25\% [3,4,7-13].Only three of our patients required revision PC due to re-stenosis, and one of them eventually also required suture lateralization and partial arrytenoidectomy in order to relieve her dyspnea. In 5 patients VFI was only a part of the etiology of dyspnea. In this subset of patients other glottic narrowing pathologies (e.g., posterior web, anterior web, edematouse false vocal fold) were surgically addressed simultaneously with PC for VFI in order to achieve a wide glottic inlet. In these patients the respiratory relief should be contributed to the combination of PC and other glottic-widening procedures.

In conclusion, a PC is an easy, safe, and effective procedure for tracheostomy weaning and respiratory relief in patients with VFI. A revision PC may be indicated in some patients. A breathy voice should be expected, and a few patients will experience dysphagia to fluids that may be addressed by instructing the patient to use a fluid thickener and take small sips.

\section{Acknowledgement}

Esther Eshkol is thanked for editorial assistance.

\section{References}

1. Brake MK, Anderson J. Bilateral vocal fold immobility: a 13 year review of etiologies, management and the utility of the Empey index. J Otolaryngol Head Neck Surg 2015;44:1-7.

2. Hashmi NK, Ransom E, Nardone H, Redding N, Mirza N. Quality of life and self-image in patients undergoing tracheostomy. Laryngoscope 2010;120:S196.

3. Benninger MS, Gillen JB, Altman JS. Changing etiology of vocal fold immobility. Laryngoscope 1998;108:1346-50.

4. Pinto JA, Godoy LB, Marquis VW, Sonego TB, Leal Cde F. Bilateral vocal fold immobility: diagnosis and treatment. Braz J Otorhinolaryngol 2010;7777:594-9.

5. Shiki N, Olamura M, Tanabe M, Morimoto M. Differential diagnosis of hoarseness. Folia Phoniatr (Basel) 1969;21:9-23.

6. Hirano M. Clinical examination of voice. New York: Springer; 1981.

7. Segas SJ, Stavroulakis P, Manolopoulos LM, Yiotakis J, Adamopoulos G. Management of bilateral vocal fold paralysis: Experience at the University of Athens. Otolaryngol Head Neck Surg 124:1:68-71.

8. Dennis DP, Kashima H. Carbon dioxide laser posterior cordectomy for treatment of bilateral vocal cord paralysis. Ann Otol Rhinol Laryngol 1989;98:930-4.

9. Landa M, Luqui I, Gómez J, Martínez Z. Posterior cordectomy. Our experience. Acta Otorrinolaringol Esp 2013;63:26-30.

10. Virmani N, Dabholkar J. Laser-assisted posterior cordotomy for bilateral vocal fold paralysis: our experience. J Head Neck Physicians Surg 2016;4:23-8.

11. Mawaddah A, Marina MB, Halimuddin S, Mohd Razif MY, Abdullah S. A ten-year Kuala Lumpur review on laser posterior cordectomy for bilateral vocal fold immobility. Malaysian J Med Sci 2016;23:65-70.

12. Hillel AT, Giraldez L, Samad I, Gross J, Klein AM, Johns MM 3rd. Voice outcomes following posterior cordotomy with medial arytenoidectomy in patients with bilateral vocal fold immobility. JAMA Otolaryngol Head Neck Surg 2015;141:728-732.

13. Nawka T, Sittel C, Gugatschka M, Arens C, Lang-Roth R, Wittekindt $\mathrm{C}$, et al. Permanent transoral surgery of bilateral vocal fold paralysis: a prospective multi-center trial. Laryngoscope 2015;125:1401-8.

Received for publication: 4 December 2019. Accepted for publication: 3 March 2020.

This work is licensed under a Creative Commons Attribution-NonCommercial 4.0 International License (CC BY-NC 4.0).

CCopyright: the Author(s), 2020

Licensee PAGEPress, Italy

Multidisciplinary Respiratory Medicine 2020; 15:35

doi:10.4081/mrm.2020.35 